\title{
AVALIAÇÃO DA PRODUÇÃO DE ETANOL POR FERMENTAÇÃO EM ESTADO SÓLIDO A PARTIR DE FARELO DE ARROZ
}

\author{
N.I. CANABARRO ${ }^{1}$, C. ALESSIO ${ }^{1}$, N. SUSIN ${ }^{1}$, P.R.S. SALBEGO ${ }^{1}$, W. PRIAMO ${ }^{2}$ e M.A. \\ MAZZUTI $^{1}$ \\ 1Universidade Federal de Santa Maria, Departamento de Engenharia Química \\ ${ }^{2}$ Departmento de Tecnologia de alimentos, IFRS - Campus Sertão \\ E-mail para contato: claudianp@msn.com
}

\begin{abstract}
RESUMO - A crise energética causada pela exaustão de combustíveis fósseis e problemas ambientais tem causado preocupações em pesquisadores, fazendo com que os mesmos procurem alternativas para substituir os combustíveis fósseis por fontes renováveis. Uma alternativa interessante é o uso da fermentação em estado sólido para produção de biocombustíveis, utilizando resíduos agroindustriais como fonte de açúcares fermentáveis. A metodologia de planejamento de experimentos foi utilizada com objetivo de identificar as variáveis significativas no processo de fermentação em estado sólido, através da aplicação de um design de experimentos Plackett \& Burmann, onde foi encontrada uma produção máxima de etanol de 18,37 \%w/w. O método de superfície de resposta foi utilizado para realizar a otimização do processo, com base na avaliação de um delineamento composto central rotacional (DCCR).
\end{abstract}

\section{INTRODUÇÃO}

O consumo global de energia vem aumentando com o crescimento da população mundial e o crescimento industrial (Field, 2008). Combustíveis derivados do petróleo são muito eficientes até o momento, mas estão sendo ameaçados por não serem renováveis e por serem poluentes. Uma solução para combater o problema é o investimento em fontes limpas e renováveis, tais como biocombustíveis (Sarkar, 2012). Uma alternativa é o emprego da fermentação em estado sólido para produção de biocombustíveis utilizando resíduos agroindustriais como fonte de açúcares fermentescíveis. A biomassa lignocelulósica tem sido considerada a matéria-prima ideal para a produção de biocombustíveis, uma vez que não compete com os recursos alimentares e pode, potencialmente, reduzir a emissão de dióxido de carbono, de acordo com as pesquisas de Galbe e Zacchi (2007).

O processo de fermentação em estado sólido (SSF) é um processo em que a cultura de microrganismo se dá em matriz sólida, onde o conteúdo de líquido ligado a ela está a um nível de atividade de água que, por um lado, assegure o crescimento e metabolismo das células e por outro, não ultrapasse a capacidade máxima de ligação da água com a matriz sólida. A SSF oferece algumas vantagens em relação à fermentação submersa, como o uso de meios simples de crescimento, utilização de resíduos agroindustriais, menor quantidade de água, menor volume de efluentes, baixo consumo de energia, e facilidade de separação do produto. (Singhania, 2009). 
Porém, para realizar o processo de fermentação em estado sólido é necessário que a biomassa passe por um processo de pré tratamento, para que a glicose seja liberada para o meio fermentativo e o microorganismo a converta no produto final. Uma alternativa é a Sacarificação e fermentação simultâneas, que de acordo com os estudos de Takagi (1977) mostraram diminuição da inibição do produto final e riscos de contaminação do meio. O uso do processo de fermentação em estado sólido está sendo relatado por vários pesquisadores como uma alternativa para a fermentação submersa. Este trabalho teve como objetivo a produção de etanol através do processo de sacarificação e fermentação em estado sólido simultâneo, a partir de uma mistura de farelo e casca de arroz, utilizando Saccharomyces cerevisiae.

\section{MATERIAIS E MÉTODOS}

\subsection{Microrganismos e meio de cultura}

O microrganismo Saccharomyces cerevisiae foi disponibilizado pelo departamento de engenharia química da Universidade Federal de Santa Maria (Brasil, RS). O meio sintético para o crescimento do microrganismo foi composto por: sacarose $(20 \mathrm{~g} / \mathrm{L})$, extrato de levedura $(5 \mathrm{~g} / \mathrm{l}), \mathrm{K}_{2} \mathrm{PO}_{4}$ $(5 \mathrm{~g} / \mathrm{l}), \mathrm{NH}_{4} \mathrm{Cl}(1,5 \mathrm{~g} / \mathrm{l}), \mathrm{KCl}(1,15 \mathrm{~g} / \mathrm{l})$ e $\mathrm{MgSO}_{4} \cdot 7 \mathrm{H}_{2} \mathrm{O}(0,65 \mathrm{~g} / \mathrm{l})$, sendo o pH do meio corrigido para 6.8 utilizando $\mathrm{NaOH} 1 \mathrm{~N}$. O inóculo foi realizado em um Erlenmeyer $(250 \mathrm{~mL})$, onde foram adicionados $1 \mathrm{~g}$ de Saccharomyces cerevisiae em $100 \mathrm{~mL}$ do meio a $120 \mathrm{RPM}$ e $30^{\circ} \mathrm{C}$, por $24 \mathrm{~h}$.

\subsection{Sacarificação e Fermentação em estado sólido simultâneo (SSSF)}

Os experimentos de sacarificação e fermentação em estado sólido simultâneo foram realizados em frascos de $600 \mathrm{~mL}$, onde foram avaliadas as seguintes variáveis: Temperatura $\left(30-40^{\circ} \mathrm{C}\right)$, proporção de farelo e casca de arroz (1:1 - 1:3), percentagem de inóculo (5 - 15\%), umidade (60 $80 \%)$, porcentagem de farelo de soja e água de maceração de milho (5 - 15\%), quantidade de spirizyme fuel e celulase $(0,2-0,6 \%)$. A fim de identificar as variáveis mais significativas que afetam diretamente o processo de produção de etanol por fermentação em estado sólido, foi realizado um planejamento de experimentos Plackett \& Burmann. O design PB é um planejamento de experimentos fatorial de dois níveis e permite a investigação de $\mathrm{n}-1$ variáveis em $\mathrm{n}$ experimentos. (Plackett e Burman, 1946)

A Metodologia de superfície de resposta foi utilizada a fim de otimizar as condições do processo de produção de etanol. Um delineamento composto central rotacional (DCCR) com cinco níveis codificados foi realizado em um design de 12 experimentos com três réplicas no ponto central, com base na análise estatística realizada anteriormente no planejamento PB. 


\subsection{Extração e quantificação do Etanol}

O procedimento de extração foi realizado de acordo com razão sólido/líquido 0,3, expondo as amostras à agitação mecânica de 120 RPM e temperatura controlada $\left(30^{\circ} \mathrm{C}\right)$ por 60 minutos, utilizando um agitador mecânico Tecnal te-240. Por fim, a amostra foi retirada e filtrada em papel filtro (Qualy, $80 \mathrm{~g} / \mathrm{m}^{2}$ ) condicionadas em frascos e encaminhadas para análise de quantificação.

As análises de quantificação de etanol foram realizadas através de um analisador de teor alcóolico Alcolyzer Wine M/WE - Wine Analysis System. Este analisador efetua a leitura da concentração alcóolica resultando em uma porcentagem volume/volume (\% v/v), permitindo avaliar alíquotas cujas concentrações oscilam de $0-20 \%$ v/v e apresentando um erro de medição de aproximadamente $\pm 0,1 \%$ v/v de álcool. As análises de açúcares redutores foram realizadas através do procedimento de DNS, desenvolvido por Miller (1959).

\section{RESULTADOS E DISCUSSÃO}

\subsection{Avaliação da Produção de Etanol}

Os dados sobre a produção de etanol utilizando diferentes condições experimentais são apresentados na Tabela 1 .

Tabela 1| Variáveis e níveis de teste para o planejamento Plackett \& Burmann

\begin{tabular}{cccccccccc}
\hline Ensaio & $\mathbf{x}_{\mathbf{1}}$ & $\mathbf{x}_{\mathbf{2}}$ & $\mathbf{x}_{\mathbf{3}}$ & $\mathbf{x}_{\mathbf{4}}$ & $\mathbf{x}_{\mathbf{5}}$ & $\mathbf{x}_{\mathbf{6}}$ & $\mathbf{x}_{\mathbf{7}}$ & $\mathbf{x}_{\mathbf{8}}$ & $\begin{array}{c}\text { Etoh } \\
(\boldsymbol{\%} \mathbf{w} / \mathbf{w})\end{array}$ \\
\hline 1 & $40(+1)$ & $01: 01(+1)$ & $5(-1)$ & $60(-1)$ & $15(+1)$ & $5(-1)$ & $0,2(-1)$ & $0,6(+1)$ & 1,46 \\
2 & $40(+1)$ & $01: 03(-1)$ & $5(-1)$ & $60(-1)$ & $5(-1)$ & $15(+1)$ & $0,2(-1)$ & $0,2(-1)$ & 3,54 \\
3 & $40(+1)$ & $01: 03(-1)$ & $15(+1)$ & $60(-1)$ & $5(-1)$ & $5(-1)$ & $0,6(+1)$ & $0,2(-1)$ & 5,91 \\
4 & $40(+1)$ & $01: 01(+1)$ & $15(+1)$ & $80(+1)$ & $5(-1)$ & $5(-1)$ & $0,2(-1)$ & $0,6(+1)$ & 2,05 \\
5 & $30(-1)$ & $01: 01(+1)$ & $15(+1)$ & $80(+1)$ & $15(+1)$ & $5(-1)$ & $0,2(-1)$ & $0,2(-1)$ & 4,07 \\
6 & $40(+1)$ & $01: 03(-1)$ & $15(+1)$ & $80(+1)$ & $15(+1)$ & $15(+1)$ & $0,2(-1)$ & $0,2(-1)$ & 8,86 \\
7 & $30(-1)$ & $01: 01(+1)$ & $5(-1)$ & $80(+1)$ & $15(+1)$ & $15(+1)$ & $0,6(+1)$ & $0,2(-1)$ & 6,36 \\
8 & $40(+1)$ & $01: 03(-1)$ & $15(+1)$ & $60(-1)$ & $15(+1)$ & $15(+1)$ & $0,6(+1)$ & $0,6(+1)$ & 13,39 \\
9 & $40(+1)$ & $01: 03(-1)$ & $5(-1)$ & $80(+1)$ & $5(-1)$ & $15(+1)$ & $0,6(+1)$ & $0,6(+1)$ & 6,60 \\
10 & $30(-1)$ & $01: 03(-1)$ & $15(+1)$ & $60(-1)$ & $15(+1)$ & $5(-1)$ & $0,6(+1)$ & $0,6(+1)$ & 29,12 \\
11 & $30(-1)$ & $01: 01(+1)$ & $15(+1)$ & $80(+1)$ & $5(-1)$ & $15(+1)$ & $0,2(-1)$ & $0,6(+1)$ & 36,73 \\
12 & $40(+1)$ & $01: 01(+1)$ & $5(-1)$ & $80(+1)$ & $15(+1)$ & $5(-1)$ & $0,6(+1)$ & $0,2(-1)$ & 17,35 \\
13 & $30(-1)$ & $01: 03(-1)$ & $5(-1)$ & $60(-1)$ & $15(+1)$ & $15(+1)$ & $0,2(-1)$ & $0,6(+1)$ & 8,67 \\
14 & $30(-1)$ & $01: 01(+1)$ & $15(+1)$ & $60(-1)$ & $5(-1)$ & $15(+1)$ & $0,6(+1)$ & $0,2(-1)$ & 19,95 \\
15 & $30(-1)$ & $01: 01(+1)$ & $5(-1)$ & $80(+1)$ & $5(-1)$ & $5(-1)$ & $0,6(+1)$ & $0,6(+1)$ & 22,01 \\
16 & $30(-1)$ & $01: 01(+1)$ & $5(-1)$ & $60(-1)$ & $5(-1)$ & $5(-1)$ & $0,2(-1)$ & $0,2(-1)$ & 9,43 \\
17 & $35(0)$ & $01: 02(0)$ & $10(0)$ & $70(0)$ & $10(0)$ & $10(0)$ & $0,4(0)$ & $0,4(0)$ & 18,98 \\
18 & $35(0)$ & $01: 02(0)$ & $10(0)$ & $70(0)$ & $10(0)$ & $10(0)$ & $0,4(0)$ & $0,4(0)$ & 19,34 \\
19 & $35(0)$ & $01: 02(0)$ & $10(0)$ & $70(0)$ & $10(0)$ & $10(0)$ & $0,4(0)$ & $0,4(0)$ & 17,79
\end{tabular}

Onde: X1 Temperatura, X2 proporção farelo/casca de arroz, X3 inóculo (\%), X4 umidade (\%), X5 farelo de soja (\%w/w), X6 água de maceração de milho (\%w/w), X7 Spirzyme Fuel (\%v/w), X8 celulase (\%) v/w. 


\section{9 a 22 de outubro de 2014 \\ Florianópolis/SC}

Com base nos dados apresentados na Tabela 1 observa-se que a maior produção de etanol é encontrada no experimento 11 , onde se obteve $18,37 \% \mathrm{w} / \mathrm{w}$, em concordância no que foi encontrado no trabalho de Li et al (2013) que encontraram 16\% w/w de etanol em um processo de fermentação em estado sólido utilizando talos de sorgo sacarino. As análises estatísticas foram realizadas usando o software estatístico STATISTICA7 e os resultados encontrados para o planejamento experimental Plackett \& Burmann são apresentados na Tabela 2.

Tabela 2| Efeito estimado, coeficiente de regressão e valores de t e p para o Plackett \& Burmann

\begin{tabular}{cccccc} 
Variáveis & Efeito & Erro Padrão & $\mathbf{t}$ & $\mathbf{p}$ & Coeficientes \\
\hline Média & 0,490526 & 0,058880 & 8,33100 & 0,000008 & 0,490526 \\
Temperatura & 0,038750 & 0,128325 & 0,30197 & 0,768864 & 0,019375 \\
FC & 0,193750 & 0,128325 & 1,50984 & 0,162017 & 0,096875 \\
Inóculo (\%) & $-0,108750$ & 0,128325 & $-0,84746$ & 0,416563 & $-0,054375$ \\
U (\%) & 0,516250 & 0,128325 & 4,02298 & 0,002427 & 0,258125 \\
Farelo de soja (\%) & $-0,156250$ & 0,128325 & $-1,21761$ & 0,251324 & $-0,078125$ \\
AMM (\%) & 0,121250 & 0,128325 & 0,94486 & 0,366998 & 0,060625 \\
Spirizyme fuel (\%) & $-0,068750$ & 0,128325 & $-0,53575$ & 0,603843 & $-0,034375$ \\
Celulase (\%) & $-0,148750$ & 0,128325 & $-1,15916$ & 0,273320 & $-0,074375$ \\
\hline
\end{tabular}

Onde: FC é a proporção de farelo e casca de arroz e U é a umidade no meio fermentativo.

De acordo com os estudos realizados por Ferreira (2009) e com base nos resultados apresentados na Tabela 2, é possível afirmar que a umidade é um parâmetro significativo para a produção de etanol por fermentação em estado sólido com $90 \%$ de confiança, sendo fundamental para o processo de fermentação em estado sólido por manter a atividade microbiana no meio fermentativo. Os demais parâmetros não demonstraram serem significativos no processo, porém vale ressaltar que a importância da concentração de substrato está diretamente ligada ao processo industrial, onde o processo envolve grandes volumes de substrato e onde haverá uma dificuldade maior na dissipação do calor metabólico, inibição pelo substrato e o empacotamento do leito fermentativo em função do tamanho das partículas utilizadas. Sendo assim, a proporção de substrato foi tomada como significativa no processo. As fontes de nitrogênio (farelo de soja e água de maceração de milho) não apresentaram significância relevante no processo, mostrando que o substrato utilizado fornece suplementação suficiente para que o processo se desenvolva normalmente, reduzindo assim os gastos com o mesmo. As enzimas responsáveis pela hidrólise da biomassa, liberando as pentoses e hexoses para o consumo do microrganismo, de certa forma são importantes para o processo, pois sacarificam o meio fermentativo, porém através da análise estatística as mesmas não se mostraram significantes. 


\subsection{Metodologia de Superfície de Resposta (RSM)}

RSM foi empregada para maximizar a produção de etanol. Duas variáveis, umidade e proporção de substrato foram avaliadas no Delineamento Composto Central Rotacional (CCRD), conforme analisado no planejamento anterior. Doze ensaios foram realizados para localizar as condições ótimas para a produção máxima de etanol e os resultados estão apresentados na Tabela 3.

Tabela 3| Matriz CCRD e resultados

\begin{tabular}{cccc}
\hline Ensaio & $\mathbf{X}_{\mathbf{1}}$ & $\mathbf{X}_{\mathbf{2}}$ & EtOH $(\boldsymbol{\%} \mathbf{w} / \mathbf{w})$ \\
\hline 1 & $1: 1(-1)$ & $35(-1)$ & 19,36 \\
2 & $1: 1(-1)$ & $65(1)$ & 16,48 \\
3 & $3: 1(1)$ & $35(-1)$ & 26,68 \\
4 & $3: 1(1)$ & $65(1)$ & 22,24 \\
5 & $2: 1(0)$ & $75(0)$ & 17,52 \\
6 & $2: 1(0)$ & $75(0)$ & 22,49 \\
7 & $1,5: 1(-1,41)$ & $75(0)$ & 18,57 \\
8 & $4: 1(1,41)$ & $75(0)$ & 18,05 \\
9 & $2: 1(0)$ & $60(-1,41)$ & 21,45 \\
10 & $2: 1(0)$ & $90(1,41)$ & 27,20 \\
1 & $2: 1(0)$ & $75(0)$ & 25,11 \\
\end{tabular}

Onde: $\mathrm{X}_{1}$ proporção farelo/casca de arroz e $\mathrm{X}_{2}$ umidade (\%).

Variáveis fixadas: $\mathrm{T}=37^{\circ} \mathrm{C}, \mathrm{AMM}=5 \%, \mathrm{FS}=5 \%$, Celulase $=0,6 \% \mathrm{v} / \mathrm{v}, \mathrm{S}$. Fuel $=0,2 \% \mathrm{v} / \mathrm{v}$.

Conforme pode ser observado na Tabela 4, a maior produção de etanol foi obtida no experimento 12, onde foram produzidos $14,39 \% \mathrm{w} / \mathrm{w}$ de etanol. A Tabela 4 apresenta as análises realizadas através do CCRD.

Tabela 4| Efeito estimado, coeficiente de regressão e valores de t e p para o CCRD

\begin{tabular}{cccccc}
\hline Fatores & Efeito & Erro Padrão & t & p & Coeficientes \\
\hline Média & 1,025000 & 0,050363 & 20,35234 & 0,000001 & 1,025000 \\
FC (L) & 0,117929 & 0,071224 & 1,65575 & 0,148851 & 0,058964 \\
FC (Q) & $-0,281250$ & 0,079631 & $-3,53194$ & 0,012339 & $-0,140625$ \\
U (L) & $-0,123033$ & 0,071224 & $-1,72742$ & 0,134835 & $-0,061517$ \\
U (Q) & $-0,236250$ & 0,079631 & $-2,96683$ & 0,025060 & $-0,118125$ \\
FC x U & $-0,030000$ & 0,100726 & $-0,29784$ & 0,775870 & $-0,015000$ \\
\hline
\end{tabular}

Os resultados apresentados na Tabela 4 foram usados para construir um modelo matemático expressando a produção de etanol em função das variáveis independentes. A Equação 1 mostra os termos significantes do modelo $(\mathrm{p}<0,1)$.

$\% E t O H=1,025-0,2812 F C^{2}-0,2362 U^{2}$

onde \%EtOH é a concentração de etanol na amostra, FC é a proporção de substrato (farelo e casca de arroz) no meio fermentativo e U é a umidade no meio fermentativo.

O modelo apresentado foi validado através da análise de variância (ANOVA), obtendo um F calculado 10 vezes maior que o tabelado (ANOVA), e com coeficiente de determinação ( $\mathrm{R}^{2}$ ) de 0,797 . 
Com base no modelo validado, foi realizada a otimização da produção de etanol, onde os resultados são apresentados na Figura 1.

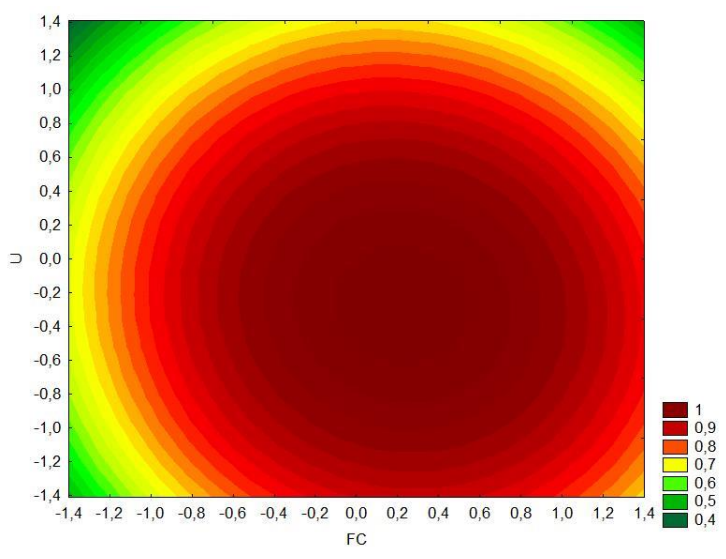

Figura 1 Superfície de contorno para a produção de etanol.

A Figura 1 apresenta a influência da umidade e da proporção de substrato na produção de etanol por sacarificação e fermentação em estado sólido simultânea. As maiores produções de etanol foram obtidas em uma faixa de umidade variando de 40 a $80 \%$ e para a proporção de substrato variando de 1:1 a 1:3. O modelo permite afirmar que existe uma região ótima na superfície de contorno, além disso, é possível afirmar que a condição otimizada foi encontrada para umidade de $75 \%$ e proporção de substrato de $2: 1$. Nesta condição, a produção máxima de etanol foi em torno de 14,39\% w/w. Baseado na condição ótima encontrada na Figura 1 foi realizado um estudo cinético para o processo, onde se avaliou a produção de etanol e o consumo do substrato na forma de açúcares redutores, conforme é apresentado na Figura 2.

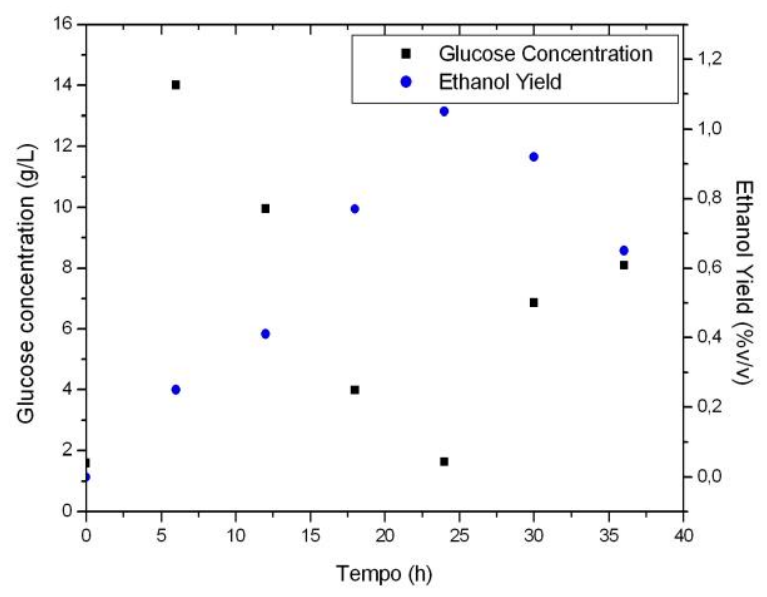

Figura 2 - Cinética do processo nas condições ótimas. 
A cinética do processo de sacarificação e fermentação simultâneo apresentado na Figura 2 mostra que a produção de etanol é inversamente proporcional ao consumo de substrato no meio. Tal comportamento é padrão em processos fermentativos onde, por lógica, o microorganismo utilizado no processo consome o substrato convertendo-o no produto de interesse e mais alguns subprodutos. No caso da Saccharomyces cerevisiae o produto de interesse é o etanol e o subproduto é basicamente dióxido de carbono. Analisando a Figura 2 observa-se que a produção máxima de etanol obtida foi no tempo de 24 horas, mesmo tempo em que o consumo máximo do substrato foi encontrado. Neste ponto a velocidade de obtenção do produto é máxima $\left(1,145 \mathrm{~g} \mathrm{~L}^{-1} \mathrm{~h}^{-1}\right)$, consumindo praticamente todo o substrato presente no meio e acarretando em uma alta conversão do mesmo no produto final de interesse. Após 24 horas observa-se um decaimento na produção de etanol que pode ser atribuída a perda de etanol para o meio, através da sua evaporação, que é consequiência do calor metabólico gerado pelo microorganismo ou pela adsorção do etanol através dos poros do farelo e da casca de arroz. Já para o substrato nota-se um aumento na sua concentração após 24 horas de processo, podendo ser atribuído pela continuidade da ação catalítica dos dois complexos enzimáticos presentes no meio fermentativo e através da desativação do microorganismo no processo, fazendo com que o consumo do substrato seja reduzido e assim aumente a concentração do mesmo no meio fermentativo.

De maneira adversa aos processos de hidrólise e fermentação tradicionais, o processo de sacarificação e fermentação simultâneo deve operar com temperaturas e tempos que atendam ambos os agentes atuantes no meio, neste caso, o microorganismo e os complexos enzimáticos. O comportamento apresentado após 24 horas de processo esboça perfeitamente a dificuldade encontrada no processo. Neste momento e nas condições propostas $\left(37^{\circ} \mathrm{C}\right.$ e 24 horas $)$ o microorganismo se encontra em condições ótimas para seu desenvolvimento no meio fermentativo, diferente dos complexos enzimáticos, que têm sua atividade catalítica máxima em temperaturas mais elevadas, em torno de $50{ }^{\circ} \mathrm{C}$, conforme Singh et al (2014), que realizaram a hidrólise enzimática da casca de arroz utilizando a mesma temperatura. Da mesma maneira, os tempos dos processos diferem entre si, onde para o processo de fermentação a produção máxima é alcançada em 24 horas e para a hidrólise geralmente o tempo de processo é mais elevado, superando as 24 horas do processo fermentativo. Assim, como mostrado na Figura 2, os complexos enzimáticos levarão mais tempo para realizar a hidrólise, fazendo com que ocorra o decaimento da concentração de substrato no meio através do seu consumo pelo microrganismo até o tempo de 24 horas e posteriormente um aumento de sua concentração como consequência da continuidade da ação catalítica das enzimas presentes no processo.

\section{CONCLUSÃO}

Neste trabalho foi realizado a otimização do processo de fermentação em estado sólido para a produção de etanol de segunda geração. O meio de cultura foi composto principalmente de farelo e casca de arroz, sendo suplementado com água de maceração de milho e farelo de soja. Os resultados obtidos mostraram que a produção de etanol por fermentação em estado sólido pode ser uma alternativa viável frente aos processos tradicionais utilizados na fabricação do mesmo, como no caso da fermentação submersa. A maior produção de etanol foi 18,37 \%w/w, sendo as condições ótimas para a produção do mesmo $75 \%$ de umidade e proporção de substrato de $2: 1$. 


\section{REFERÊNCIAS}

Ferreira, S.; Duarte, A.P.; Ribeiro, M.H.L.; Queiroz, J.A.; Domingues, F.C. Response surface optimization of enzymatic hydrolysis of Cistus ladaniferand Cytisus striatusfor bioethanol production. Biochem. Eng J, v 45, p 192-200, 2009.

Field, C.B.; Campbell, J.E.; Lobell, D.B. Biomass energy: the scale of the potential resource. Trends Ecol Evol, v 23, p 65-72, 2008.

Galbe, M.; Zacchi, G. Pretreatment of lignocellulosic materials for efficient bioethanol production. Adv Biochem Eng/Biotechnol, v 108, p 41-65, 2007.

Li, S.; Li, G.; Zhou, L.; Han, B.; Hou, W.; Wang J.; Tiancheng, L. A demonstration study of ethanol production from sweet sorghum stems with advanced solid state fermentation technology. App Energy, v 102, p 260-265, 2013.

Miller, G L. Use of Dinitrosalicylic Acid Reagent for Determination of Reducing Sugar. Pioneering Research Division, Quarfermasfer Research and Engineering Center, Natick, Mass, 1959.

Plackett, R.L.; Burman, J.P. The design of optimum multifactorial experiments. Biometrika, v 33, p 305-325, 1946.

Saha, B.C.; Nichols, N.N.; Qureshi, N.; Cotta, M.A. Comparison of separate hydrolysis and fermentation and simultaneous saccharification and fermentation processes for ethanol production from wheat straw by recombinant Escherichia coli strain FBR5. App Microbio. Biotechnol, v 92, p 865-874, 2011.

Sarkar, N.; Ghosh, S.K.; Bannerjee, S.; Aikat, K. Bioethanol production from agricultural wastes: an overview, Renew. Energy, v. 37, p 19-27, 2012.

Singhania, R.R.; Patel, A.K.; Soccol, C.R.; Pandey, A. Recent advances in solid-state fermentation. Biochem Eng J, v 44, p 13-18, 2009.

Singh, A.; Bajar, S.; Bishnoi, N. Enzymatic hydrolysis of microwave alkali pretreated rice husk for ethanol production by Saccharomyces cerevisiae, Scheffersomyces stipitis and their coculture. Fuel, v 116, p 699-702, 2014.

Takagi, M.; Abe, S.; Suzuki, S.; Emert, G.H.; Yata, N. A method for production of alcohol directly from cellulose using cellulase and yeast, in: Proceedings of the Bioconversion Symposium, IIT, Delhi, p. 551-571, 1977. 